\title{
Stable half-metallic ferromagnetism in nonstoichiometric cubic bi- nary chromium chalcogenides
}

\author{
SAN-DONG GuO and BANG-Gui LiU ${ }^{(a)}$ \\ Institute of Physics, Chinese Academy of Sciences, Beijing 100190, China \\ Beijing National Laboratory for Condensed Matter Physics, Beijing 100190, China \\ PACS 75.30.-m - Intrinsic properties of magnetically ordered materials \\ PACS 75.10.-b - General theory and models of magnetic ordering \\ PACS 75.90.+w - Other topics in magnetic properties and materials
}

\begin{abstract}
We find that three nonstoichiometric cubic binary chromium chalcogenides, namely $\mathrm{Cr}_{3} \mathrm{~S}_{4}, \mathrm{Cr}_{3} \mathrm{Se}_{4}$, and $\mathrm{Cr}_{3} \mathrm{Te}_{4}$, are stable half-metallic ferromagnets with wide half-metallic gaps on the basis of systematic state-of-the-arts first-principles calculations. We optimize their structures, and then calculate their magnetic moments, electronic structures, formation heats, and elastic moduli and investigate their structural stability and robustness of ferromagnetism against antiferromagnetic fluctuations. Our calculated results show that the three sulvanite phases are structurally stable and ferromagnetically robust, and hence could be realized as epitaxial thin films. We attribute the structural and ferromagnetic stability and the better half-metallicity to their special effective Cr valence 2.667+. These findings will open doors for much more highperformance spintronic materials compatible with current semiconductor technology.
\end{abstract}

It is believed that next-generation high-performance computers can be achieved through using the spin freedom of electron in key materials and devices of current semiconductor technology $[1,2]$. The half-metallic ferromagnet, first discovered in NiMnSb by de Groot et al in 1983, has almost $100 \%$ spin polarization near the Fermi level [3-5]. This feature of half-metallic ferromagnetism makes carriers have high spin-polarization near the Fermi level and avoid some spin-related scattering processes that should exist otherwise. These are essential to practical spintronic applications $[1,2,5]$. Halfmetallic ferromagnetism has been found in many materials, such as Heusler alloys $[3,4,6]$, transition-metal oxides [7-9], and even graphene nanoribbons under electric field [10]. The half-metallic ferromagnetic materials compatible with semiconductor technology is believed to be promising candidates for achieving more powerful computers. For this purpose, many zincblende transition-metal pnictides and chalcogenides [11-18], regularly-doped semiconductors [19,20], semiconductor super-structures [21,22], and more related compounds have been proved to be half-metallic. Although great advance has been achieved, better half-metallic ferromagnets compatible with cur-

(a) E-mail: bgliu@mail.iphy.ac.cn rent semiconductor technology are highly desirable for the next-generation computers.

Here we find three stable half-metallic ferromagnets, namely $\mathrm{Cr}_{3} \mathrm{~S}_{4}, \mathrm{Cr}_{3} \mathrm{Se}_{4}$, and $\mathrm{Cr}_{3} \mathrm{Te}_{4}$, among nonstoichiometric cubic binary transition-metal chalcogenides on the basis of state-of-the-arts first-principles calculations. We investigate their structural stability and ferromagnetic robustness against antiferromagnetic fluctuations. Our calculated results show that the three nonstoichiometric sulvanite phases have formation heats of down to $-0.302 \mathrm{eV}$ per formula unit with respect to corresponding zincblende phases and their half-metallic gaps can be up to $1.05 \mathrm{eV}$. We attribute the structural and ferromagnetic stability and the better half-metallicity to their special effective $\mathrm{Cr}$ chemical valence $2.667+$, compared with $2+$ in the stoichiometric structures. These nonstoichiometric cubic materials have much better features for spintronic applications based on semiconductors. More detailed results will be presented in the following.

We use the full-potential linearized augmented-planewaves method within the density functional theory (DFT) [23], as implemented in package WIEN2k [24,25]. The generalized gradient approximation (GGA) [26] is used for the exchange-correlation potential. Full relativistic effects are calculated with the Dirac equations for core states, 


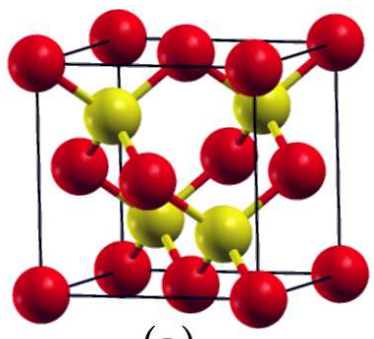

(a)

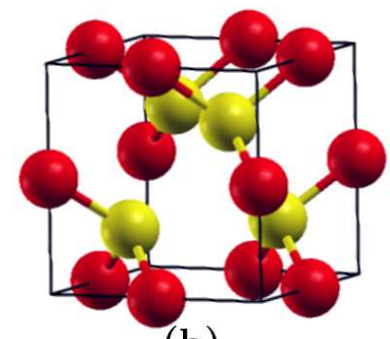

(b)
Fig. 1: (color online). The crystal structures of zincblende $\operatorname{Cr} X$ (a) and sulvanite $\mathrm{Cr}_{3} X_{4}$ (b) phases, where $X$ can be S, Se, or Te. The red (or black) ball denotes a $\mathrm{Cr}$ atom and the yellow (or gray) an $X$ atom.

and the scalar relativistic approximation is used for valence states. The spin-orbit coupling is neglected because it has little effect on our results. We use $2000 \mathrm{k}$ points in the first Brillouin zone, make the harmonic expansion up to $l_{\max }=10$ in atomic spheres, and set $R_{\mathrm{mt}} \times K_{\max }$ to 7.5. The radii of the atomic spheres of $\mathrm{Cr}$ and others are chosen so that as high accuracy as possible is obtained. The volumes are optimized in terms of total energy, and the internal position parameters with a force standard of $3 \mathrm{mRy} /$ a.u. The simplest antiferromagnetic structures are constructed by doubling the unit cells along the [100] and [110] directions. There are six $\mathrm{Cr}$ atoms in each of these doubled cells. We let three of the six Cr spins orient up and the other three down. All the spin values of the $\mathrm{Cr}$, $\mathrm{S}, \mathrm{Se}$, and Te atoms are determined naturally by the selfconsistent calculations. The structural stability is investigated through deforming the structures (with the volumes fixed) along the three directions. The elastic moduli are calculated with the standard method implemented in WIEN2k $[24,25]$. The formation heats of the cubic sulvanite structures are calculated with respect to the crystalline $\mathrm{Cr}$ phase and the corresponding zincblende structures. The self-consistent calculations are considered to be converged only when the absolute integrated chargedensity difference per formula unit between the two successive loops is less than $0.0001|e|$, where $e$ is the electron charge.

The ground-state phases for three stoichiometric $\operatorname{Cr} X$ ( $X=\mathrm{S}$, Se, and $\mathrm{Te}$ ) compounds are of nickel-arsenide (na) structure, but the zincblende (zb) structures of the $\operatorname{Cr} X$ have been shown to be higher only by $0.28-0.36 \mathrm{eV}$ per formula unit than the corresponding na ones [15], and therefore have very good stability. This actually stimulated experimental synthesis of zb-CrTe epitaxial thin films with a thickness up to $100 \mathrm{~nm}$ [16]. The sulvanite structure of $\mathrm{Cr}_{3} X_{4}$ still has cubic symmetry with space group No. 215. Each of the cations has four anionic neighbors, but each of the anions three cationic neighbors. With the cations being at the $\left(\frac{1}{2}, 0,0\right)$ sites, the anions occupy the $\left(z_{X}, z_{X}, z_{X}\right)$ sites. The two cubic structures are shown in Fig. 1. For all the sulvanite $\mathrm{Cr}_{3} X_{4}$ compounds, the
Table 1: The lattice constants $a$, the internal atomic parameters $z_{X}$, and the Cr- $X$ bond lengths $l_{v}$ of the three sulvanite $\mathrm{Cr}_{3} X_{4}$ phases. Presented in parentheses are those of the corresponding zb-Cr $X$ phases.

\begin{tabular}{cccc}
\hline \hline Name & $\mathrm{Cr}_{3} \mathrm{~S}_{4}(\mathrm{CrS})$ & $\mathrm{Cr}_{3} \mathrm{Se}_{4}(\mathrm{CrSe})$ & $\mathrm{Cr}_{3} \mathrm{Te}_{4}(\mathrm{CrTe})$ \\
\hline$a(\AA)$ & $5.344(5.469)$ & $5.679(5.833)$ & $6.117(6.292)$ \\
$z_{X}$ & $0.250(0.25)$ & $0.250(0.25)$ & $0.260(0.25)$ \\
$l_{v}(\AA)$ & $2.315(2.368)$ & $2.460(2.526)$ & $2.686(2.725)$ \\
\hline \hline
\end{tabular}
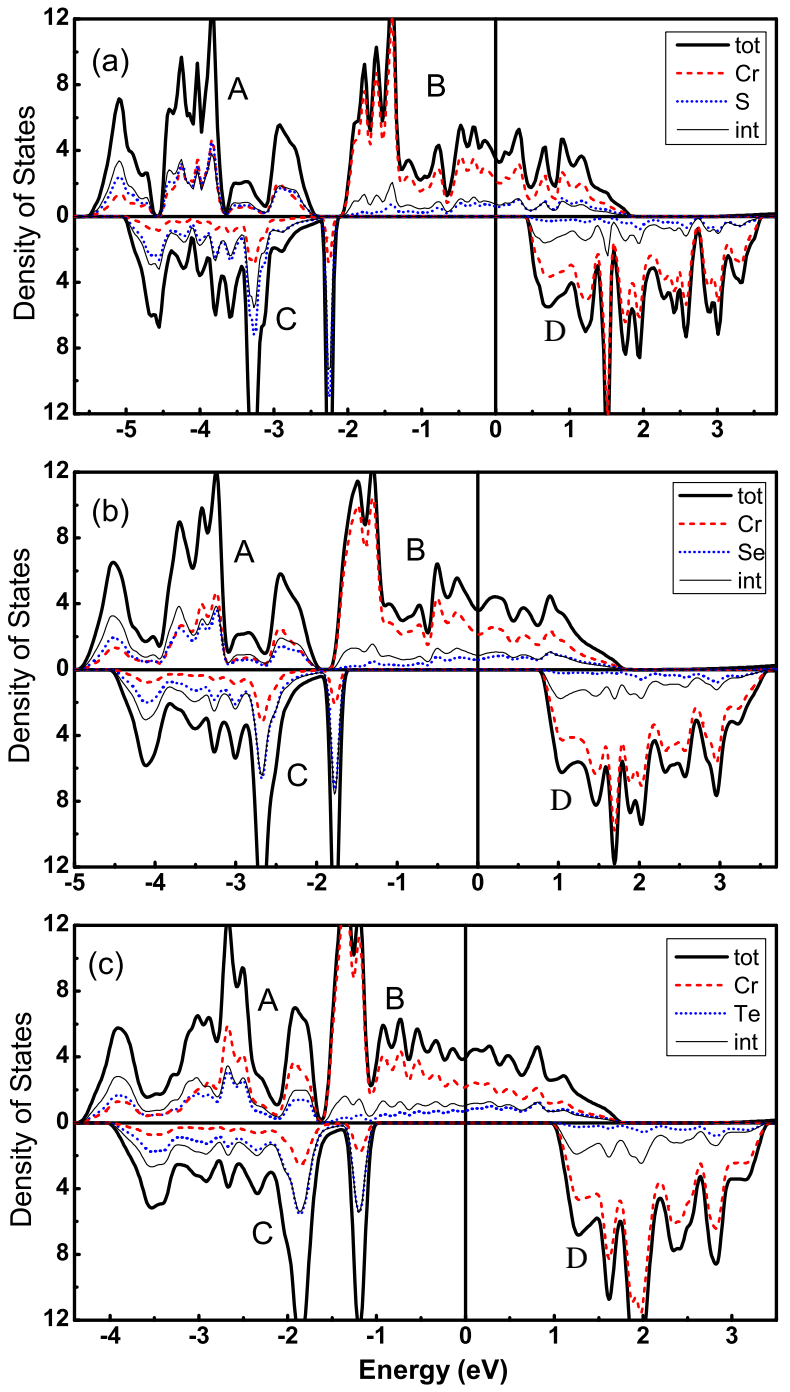

Fig. 2: (color online). Spin-dependent densities of states (DOSs) of the three sulvanite $\mathrm{Cr}_{3} X_{4}$ phases for $X=\mathrm{S}$ (a), Se (b), and Te (c). The upper part in each of the three panels is the majority-spin DOS and the lower part the minority spin. The solid thick line represents the total DOS; the red (or gray) dash, blue (or light gray) dot, and black thin lines describe the partial DOSs projected in $\mathrm{Cr}$ and $X$ atom spheres and the interstitial region, respectively. 
Stable half-metallic ferromagnetism in chromium chalcogenides

lattice constant $a$ and the internal structural parameter $z_{X}$ are optimized fully in terms of usual total energy and force standards, and then the $\mathrm{Cr}-X$ bond lengths $l_{v}$ are calculated with the optimized structures. We present our calculated $a, z_{X}$, and $l_{v}$ results for all the three sulvanite $\mathrm{Cr}_{3} X_{4}$ in Table 1 . Those of the corresponding zb $\mathrm{Cr} X$ phases are shown in parentheses for comparison. $\mathrm{Cr}_{3} \mathrm{~S}_{4}$ and $\mathrm{Cr}_{3} \mathrm{Se}_{4}$ almost keep the same $z_{X}=0.25$ as zb-CrS and zb-CrSe, and $\mathrm{Cr}_{3} \mathrm{Te}_{4}$ has $z_{X}=0.260$, a little larger than that of zb-CrTe. The sulvanite structures have smaller lattice constants than the $\mathrm{zb}$ ones by $1.6-2.6 \%$ and smaller Cr- $X$ bond lengths by 1.4-2.6\%. In the following, spindependent densities of states (DOSs), energy bands, and charge and moment density distributions are calculated in terms of the optimized structures.
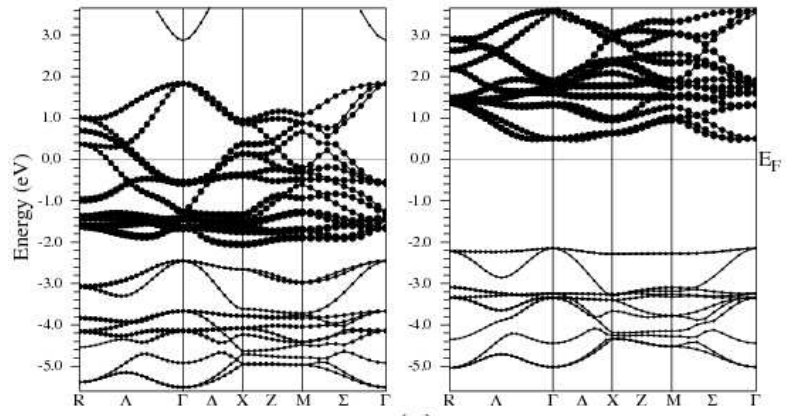

(a)
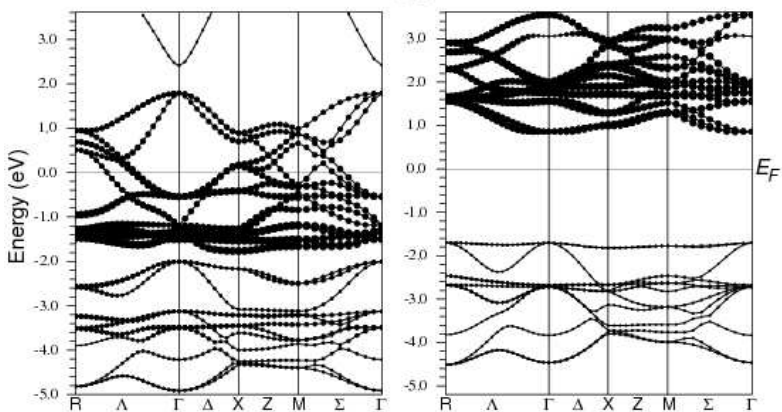

(b)
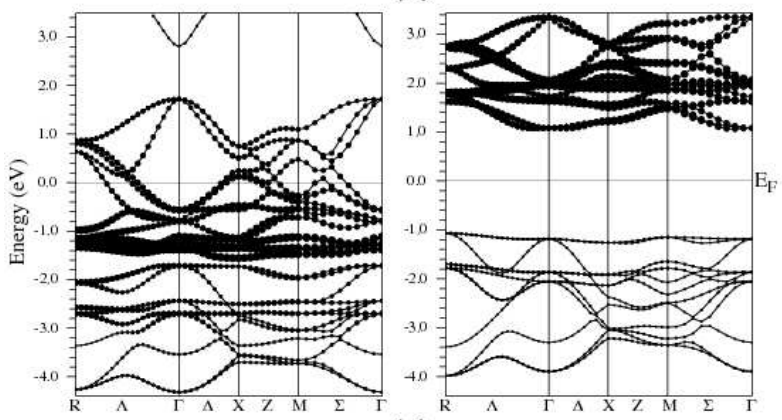

(c)

Fig. 3: Spin-dependent energy band structures (EBs) of the three sulvanite $\mathrm{Cr}_{3} X_{4}$ phases for $X=\mathrm{S}$ (a), Se (b), and Te (c). The left part in each of the three cases is the majorityspin bands and the right part the minority spin. The solid line with dots describes the EB structure along the high-symmetry points and the dot diameter is proportional to the $\mathrm{Cr} d$ weight at that point.
The spin-dependent DOSs of the three sulvanite $\mathrm{Cr}_{3} X_{4}$ $(X=\mathrm{S}, \mathrm{Se}$, and $\mathrm{Te}$ ) phases are presented in Fig. 2. For the convenience of description, we label the four different sets of the energy bands as A, B, C, and D, respectively. For each of the three $X$ cases, there is a narrow gap between $\mathrm{A}$ and $\mathrm{B}$ in the majority-spin (MAS) channel and a wide gap between $\mathrm{C}$ and $\mathrm{D}$ in the minority-spin (MIS). It is clear that there is a gap across the Fermi level in the MIS channel for each of all the three cases and therefore all the three are half-metallic ferromagnets. In addition to the total DOS, partial DOSs projected in the muffin-tin spheres of $\mathrm{Cr}$ and $X$ atoms and in the interstitial region are presented too. It is clear that the $\mathrm{B}$ and $\mathrm{D}$ bands are originated from $\mathrm{Cr}-\mathrm{d}$ states and the $\mathrm{A}$ and $\mathrm{C}$ bands mainly have the $X$-p (S, Se, or Te) character.

Presented in Fig. 3 are the spin-dependent energy bands, according to the corresponding DOS plots in Fig. 2 , for $X=\mathrm{S}$, Se, and Te. There are twelve bands in both A and C. They result from the fact that we have four $X$ ( $\mathrm{S}, \mathrm{Se}$, or Te) atoms in the sulvanite $\mathrm{Cr}_{3} X_{4}$ unit cell and each of the four has three $\mathrm{p}$ orbitals. There are fifteen bands in both $\mathrm{B}$ and $\mathrm{D}$ because we have three $\mathrm{Cr}$ atoms here and each of the three has five d orbitals. The $e_{g}$ bands are lower than the $t_{2 g}$ ones. The Cr-s state is put to higher energy than the B and D bands in both of the MAS and MIS channels. This is in contrast with zb-Cr $X$ phases whose Cr-s states are merged with the Cr-d $t_{2 g}$ states. We have also calculated charge and spin densities in three typical planes. Our results show that the charge density from the $\mathrm{A}$ and $\mathrm{C}$ bands is distributed mainly in the neighborhoods of the $X$ and $\mathrm{Cr}$ atoms, but the spin density is almost limited to $\mathrm{Cr}$ and $X$ atoms only. The filled part of the B bands are substantially localized near $\mathrm{Cr}$ and $X$ atoms. When $X$ changes from $\mathrm{S}$ to Te, the $X$ $\mathrm{p}$ character becomes less and less in the electron density from the filled $\mathrm{B}$ bands because the ionic radius becomes larger and larger.

Table 2: The magnetic moments $(M)$, half-metallic gaps $\left(G_{\mathrm{HM}}\right)$, magnetic energy differences $\left(\Delta_{E}\right)$, formation heats $\left(H_{\text {Form }}\right)$, and the elastic moduli $\left(B, C^{\prime}\right.$, and $\left.C_{44}\right)$ of the three $\mathrm{Cr}_{3} X_{4}$ phases, compared with the $\mathrm{zb}-\mathrm{Cr} X$ phases (in parentheses).

\begin{tabular}{cccc}
\hline \hline Name & $\mathrm{Cr}_{3} \mathrm{~S}_{4}(\mathrm{CrS})$ & $\mathrm{Cr}_{3} \mathrm{Se}_{4}(\mathrm{CrSe})$ & $\mathrm{Cr}_{3} \mathrm{Te}_{4}(\mathrm{CrTe})$ \\
\hline$M\left(\mu_{B}\right)$ & $10(4)$ & $10(4)$ & $10(4)$ \\
$G_{\mathrm{HM}}(\mathrm{eV})$ & $0.50(0.07)$ & $0.83(0.61)$ & $1.05(1.00)$ \\
$\Delta_{E}(\mathrm{eV})$ & 0.57 & 0.56 & 0.11 \\
$H_{\mathrm{Form}}(\mathrm{eV})$ & -0.148 & -0.114 & -0.302 \\
$B(\mathrm{GPa})$ & $72.9(63.2)$ & $57.7(59.5)$ & $44.1(45.9)$ \\
$C^{\prime}(\mathrm{GPa})$ & 8.8 & $5.9(5.6)$ & $4.8(5.5)$ \\
$C_{44}(\mathrm{GPa})$ & 34.2 & $35.9(50.7)$ & $32.9(36.4)$ \\
\hline \hline
\end{tabular}

We summarize in Table 2 the total moments per formula unit $(M)$, half-metallic gaps $\left(G_{\mathrm{HM}}\right)$ [27], and the energy differences per formula unit $\left(\Delta_{E}=E_{\mathrm{AF}}-E_{\mathrm{FM}}\right)$ 
for all the three sulvanite $\mathrm{Cr}_{3} X_{4}$ phases $(X=\mathrm{S}$, Se, and $\mathrm{Te}$ ), where $E_{\mathrm{AF}}$ and $E_{\mathrm{FM}}$ are the total energies for the $\mathrm{AF}$ and FM spin configurations. Here we define $G_{\mathrm{HM}}$ as the smaller of $E_{c b}$ and $E_{v t}$, where $E_{c b}$ is the bottom energy of the minority-spin conduction bands with respect to the Fermi level and $E_{v t}$ the absolute value of the top energy of the minority-spin valence bands. Our GGA $G_{\mathrm{HM}}$ values should be rough estimates for the minimal energies for spin flip excitations. We obtain $10 \mu_{B}$ for the magnetic moment per formula unit in all the three cases. This is because we have ten $\mathrm{Cr} d$ electrons to contribute to the moment after eight of the eighteen $\mathrm{Cr}$ electrons of the three $\mathrm{Cr}$ atoms are bonding with the sixteen $X$ p electrons of the four $X$ atoms. The half-metallic gaps are larger than the corresponding zb-Cr $X$ phases because the Fermi levels move toward lower energy level due to the fact that there are one less $\mathrm{Cr}$ atom for $\mathrm{Cr}_{3} X_{4}$, compared with the cubic $\mathrm{zb}$ unit cell including four $\mathrm{Cr}$ and $X$ atoms. The $\Delta_{E}$ results, from 0.11 to $0.57 \mathrm{eV}$, show that our FM solutions are very robust against possible AF fluctuations.

In addition, we present the formation heats per formula unit $\left(H_{\text {Form }}\right)$, the elastic moduli $\left(B, C^{\prime}\right.$, and $\left.C_{44}\right)$ of the the three $\mathrm{Cr}_{3} X_{4}$ phases in Table 2. We calculate the formation heats to investigate the stability of the $\mathrm{Cr}_{3} X_{4}$ with respect to the zb-Cr $X$ phases. For this reason, $H_{\text {Form }}$ is defined as $E\left(\mathrm{Cr}_{3} X_{4}\right)-4 E(\mathrm{zb}-\mathrm{Cr} X)+E(\mathrm{Cr})$, where $E(f)$ is the total energy of the formula $f$. The negative values of $H_{\text {Form }}$ for all the three cases mean that the sulvanite $\mathrm{Cr}_{3} X_{4}$ phases can be synthesized more easily than the corresponding zb phases, since zincblende $\mathrm{CrTe}$ has been synthesized in the form of epitaxial thin films [16]. The $B$ and $C_{44}$ values of the sulvanite phases are similar to those of the corresponding zincblende phases. The key tetragonal moduli, $C^{\prime}$, are 8.8, 5.9, and $4.8 \mathrm{GPa}$ for the three cases. The latter two $C^{\prime}$ values are comparable with those of the corresponding zincblende phases. Therefore, these calculated results show that the three sulvanite phases are mechanically stable.

The nonstoichiometry plays key roles in the sulvanite $\mathrm{Cr}_{3} X_{4}$. A Cr atom in the zb-CrX contributes a moment of $4 \mu_{B}$, but three $\mathrm{Cr}$ atoms yield $10 \mu_{B}$, not $12 \mu_{B}$, in the sulvanite $\mathrm{Cr}_{3} X_{4}$ because two more $\mathrm{Cr}$ electrons are needed to fill all the p orbitals of four $X$ atoms. Effectively, the average valence of a $\mathrm{Cr}$ atom is equivalent to $\frac{8}{3}+$ (higher than 2+) and the enhanced $\mathrm{Cr}$ valence makes the formation heat negative and hence enhances the stability of the $\mathrm{Cr}_{3} X_{4}$ with respect to the zb-Cr $X$. In zb-Cr $X, C r-\mathrm{d}_{t 2 g}$ states are hybridized substantially with $X$ p ones, and $\mathrm{Cr}$ $\mathrm{d}_{e g}$ are quite isolated. For the $\mathrm{Cr}_{3} X_{4}, \mathrm{Cr}-\mathrm{d}_{x^{2}-y^{2}}$ states remain isolated, and the other $\mathrm{Cr} d$ states are hybridized more strongly with the $X$ p ones, which makes $\mathrm{Cr}$ s state higher in energy.

In summary, we show that the three nonstoichiometric cubic binary chromium chalcogenides are stable halfmetallic ferromagnets with wide half-metallic gaps on the basis of systematic state-of-the-arts DFT calculations. Our calculated results indicate that the sulvanite $\mathrm{Cr}_{3} \mathrm{~S}_{4}$,
$\mathrm{Cr}_{3} \mathrm{Se}_{4}$, and $\mathrm{Cr}_{3} \mathrm{Te}_{4}$ are better than or approximately equal in both structural and ferromagnetic stability than zincblende $\mathrm{CrS}$, CrSe, and $\mathrm{CrTe}$, respectively. The structural stability against crystal deformations and the negative formation heats $(-0.114 \sim-0.302 \mathrm{eV}$ per formula unit) mean that they can be synthesized, at least as epitaxial thin films, more easily than the corresponding zincblende phases. We attribute the structural and ferromagnetic stability and the better half-metallicity to the higher $\mathrm{Cr}$ chemical valence, $2.667+$, in the sulvanite structures than $2+$ in the corresponding zincblende ones. These findings will open doors for much more high-performance spintronic materials compatible with current semiconductor technology.

$$
* * *
$$

This work is supported by Nature Science Foundation of China (Grant Nos. 10874232 and 10774180), by the Chinese Academy of Sciences (Grant No. KJCX2.YW.W095), and by Chinese Department of Science and Technology (Grant No. 2005CB623602).

\section{REFERENCES}

[1] S. A. Wolf, D. D. Awschalom, R. A. Buhrman, J. M. Daughton, S. von Molnar, M. L. Roukes, A. Y. Chtchelkanova, and D. M. Treger, Science 294 (2001) 1488.

[2] W. E. Pickett and J. S. Moodera, Phys. Today 54 (2001) 39.

[3] R. A. de Groot, F. M. Mueller, P. G. van Engen, and K. H. J. Buschow, Phys. Rev. Lett. 50 (1983) 2024.

[4] J. J. Attema, G. A. de Wijs, and R. A. de Groot, J. Phys.: Condens. Matter 19 (2007) 315212.

[5] W. E. Pickett and H. Eschrig, J. Phys.: Condens. Matter 19 (2007) 315203.

[6] E. Kisker, C. Carbone, C. F. Flipse, and E. F. Wassermann, J. Magn. Magn. Mater. 70 (1987) 21; J. S. Correa, Ch. Eibl, G. Rangelov, J. Braun, and M. Donath, Phys. Rev. B 73 (2006) 125316.

[7] K.-I. Kobayashi, T. Kimura, H. Sawada, K. Terakura, and Y. Tokura, Nature (London) 395 (1998) 677.

[8] S. M. Watts, S. Wirth, S. von Molnar, A. Barry, and J. M. D. Coey, Phys. Rev. B 61 (2000) 9621; S. Soeya, J. Hayakawa, H. Takahashi, K. Ito, C. Yamamoto, A. Kida, H. Asano, and M. Matsui, Appl. Phys. Lett. 80 (2002) 823.

[9] J. M. D. Coey, M. Viret, and S. von Molnar, Adv. Phys. 48 (1999) 167.

[10] Y. W. Son, M. L. Cohen, and S. G. Louie, Nature 444 (2006) 347.

[11] S. Sanvito and N. A. Hill, Phys. Rev. B 62 (2000) 15553.

[12] H. Akinaga, T. Manago and M. Shirai, Jpn. J. Appl. Phys., part 2, 39 (2000) L1118; M. Mizuguchi, H. Akinaga, T. Manago, K. Ono, M. Oshima, M. Shirai, M. Yuri, H. J. Lin, H. H. Hsieh, and C. T. Chen, J. Appl. Phys. 91 (2002) 7917.

[13] J. H. Zhao, F. Matsukura, K. Takamura, E. Abe, D. Chiba, and H. Ohno, Appl. Phys. Lett. 79 (2001) 2776.

[14] Y.-Q. Xu, B.-G. Liu, and D. G. Pettifor, Phys. Rev. B 66 (2002) 184435; B.-G. Liu, Phys. Rev. B 67 (2003) 172411. 
[15] W.-H. Xie, Y.-Q. Xu, B.-G. Liu, D. G. Pettifor, Phys. Rev. Lett. 91 (2003) 037204.

[16] M. G. Sreenivasan, K. L. Teo, M. B. A. Jalil, T. Liew, T. C. Chong, and A. Y. Du, IEEE Trans. Magn. 42 (2006) 2691; M. G. Sreenivasan, J. F. Bi, K. L. Teo, and T. Liew, J. Appl. Phys. 103 (2008) 043908; J.-F. Bi, M. G. Sreenivasan, K. L. Teo, and T. Liew, J. Phys. D: Appl. Phys. 41 (2008) 045002.

[17] J. F. Bi, J. H. Zhao, J. J. Deng, Y. H. Zheng, S. S. Li, X. G. Wu, and Q. J. Jia, Appl. Phys. Lett. 88 (2006) 142509.

[18] J. J. Deng, J. H. Zhao, J. F. Bi, Z. C. Niu, F. H. Yang, X. G. Wu, and H. Z. Zheng, J. Appl. Phys. 99 (2006) 093902; S. D. Li, J.-G. Duh, F. Bao, K.-X. Liu, C.-L. Kuo, X. S. Wu, L. Y. Lu, Z. G. Huang, and Y. W. Du, J. Phys. D: Appl. Phys. 41 (2008) 175004; S. D. Li, Z. J. Tian, J. L. Fang, J.-G. Duh, K.-X. Liu, Z. G. Huang, Y. H. Huang, Y. W. Du, Solid Stat. Commun. 149 (2009) 196.

[19] H. Ohno, Science 281 (1998) 951; T. Dietl, H. Ohno, F. Matsukura, J. Cibert, and D. Ferrand, Science 287 (2000) 1019.

[20] L.-J. Shi and B.-G. Liu, Phys. Rev. B 76 (2007) 115201; L.-F. Zhu and B.-G. Liu, J. Phys. D: Appl. Phys. 41 (2008) 215005; L.-J. Shi, L.-F. Zhu, Y.-H. Zhao, and B.-G. Liu, Phys. Rev. B 78 (2008) 195206.

[21] M. C. Qian, C. Y. Fong, K. Liu, W. E. Pickett, J. E. Pask, and L. H. Yang, Phys. Rev. Lett. 96 (2006) 027211.

[22] P. Mavropoulos, M. Lezaic, and S. Bluegel, Phys. Rev. B 72 (2005) 174428.

[23] P. Hohenberg and W. Kohn, Phys. Rev. 136 (1964) B864; W. Kohn and L. J. Sham, Phys. Rev. 140 (1965) A1133.

[24] P. Blaha, K. Schwarz, P. Sorantin, and S. B. Trickey, Comput. Phys. Commun. 59 (1990) 399.

[25] P. Blaha, K. Schwarz, G. K. H. Madsen, D. Kvasnicka, and J. Luitz, WIEN2k (K. Schwarz, Tech. Univ. Wien, Austria) (2001), ISBN 3-9501031-1-2.

[26] J. P. Perdew, K. Burke, and M. Ernzerhof, Phys. Rev. Lett. 77 (1996) 3865.

[27] B.-G. Liu, Half-Metallic Ferromagnetism and Stability of Transition Metal Pnictides and Chalcogenides, in: I. Galanakis and P. H. Dederichs (Eds.), Half-metallic alloys - Fundamentals and applications (Springer Berlin 2005), Lecture Notes in Physics 676 (2005) 267-291. 